Agronomía Costarricense 38(2): 55-65. ISSN:0377-9424 / 2014

www.mag.go.cr/rev_agr/index.html www.cia.ucr.ac.cr

\title{
HIPOCALCEMIA E HIPOMAGNESEMIA EN UN HATO DE VACAS HOLSTEIN, JERSEY Y GUERNSEY EN PASTOREO
}

\author{
Jorge Ml. Sánchez ${ }^{1 / *}$, Alejandro Saborio-Montero*
}

Palabras clave: Vacas lecheras; hipocalcemia; hipomagnesemia; vacas en pastoreo. Keywords: Dairy cows; hypocalcemia; hypomagnesemia; grazing cows.

Recibido: 07/05/14

\section{RESUMEN}

El objetivo de este estudio fue determinar la prevalencia de hipocalcemia e hipomagnesemia al parto en un hato constituido por vacas de las razas Holstein, Jersey y Guernsey, bajo las mismas condiciones de alimentación, ambiente y manejo. Se realizó en una finca localizada en Cartago, Costa Rica, y comprendió 152 vacas (62 Jersey, 41 Guernsey y 49 Holstein). Durante el período preparto las vacas pastorearon kikuyo (Kikuyuocloa clandestina) $(0,35 \mathrm{Ca} ; 0,31 \mathrm{Mg}$ y $3,50 \% \mathrm{~K}$ de la MS) y fueron suplementadas con $4 \mathrm{~kg} \cdot$ animal $^{-1} \cdot \mathrm{d}^{-1}$ de alimento concentrado bajo en $\mathrm{Ca}(0,22 \mathrm{Ca} ; 0,42 \mathrm{Mg}$ y $1,38 \% \mathrm{~K}$ de la MS) y $1 \mathrm{~kg}$ de heno $\left(0,4 \mathrm{Ca} ; 0,35 \mathrm{Mg}\right.$ y $1,85 \% \mathrm{~K}$ de la MS). $\mathrm{d}^{-1}$. Después del parto, las vacas fueron suplementadas con $1 \mathrm{~kg}$ de alimento concentrado $(0,9 \mathrm{Ca}$; $0,42 \mathrm{Mg}$ y $1,38 \% \mathrm{~K}$ de la MS) $/ 2,5$ a 3,0 kg de leche. Muestras de sangre fueron tomadas de los vasos coccígeos durante el periparto (parto $\pm 1 \mathrm{~d}$ ) y analizadas para $\mathrm{Ca}$ y $\mathrm{Mg}$. Los umbrales sanguíneos para definir la hipocalcemia como clínica o subclínica fueron: menos de 5,5 y 5,5 a 8,0 mg.dl ${ }^{-1}$ de Ca, respectivamente. Un valor de $1,8 \mathrm{mg} \cdot \mathrm{dl}^{-1}$ de $\mathrm{Mg}$, o menor, se estableció para clasificar a las vacas como hipomagnesémicas. Aunque el contenido de Ca sanguíneo durante el periparto no

1 Autor para correspondencia. Correo electrónico: jorge.sanchezgonzalez@ucr.ac.cr
Aceptado: 15/07/14

\begin{abstract}
Hypocalcemia and hipomagnesemia in a Holstein, Jersey and Guernsey grazing herd. The aim of this study was to determine the prevalence of hypocalcemia and hypomagnesemia in a Holstein, Jersey and Guernsey cow herd at parturition, under the same feeding, management and environmental conditions. It was conducted in a farm located in Cartago, Costa Rica, and comprised 152 cows (62 Jersey, 41 Guernsey and 49 Holstein). During the close up period the cows grazed kikuyu grass (Kikuyuocloa clandestina) $(0.35 \mathrm{Ca}, 0.31 \mathrm{Mg}$ and $3.50 \% \mathrm{~K}, \mathrm{DM})$ and were supplemented with $4 \mathrm{~kg}$.animal ${ }^{-1} \cdot \mathrm{d}^{-1}$ of a concentrate feed low in $\mathrm{Ca}(0.22 \mathrm{Ca} ; 0.42$ $\mathrm{Mg}$ and $1.38 \% \mathrm{~K}, \mathrm{DM})$ plus $1 \mathrm{~kg}$ of hay $(0.4 \mathrm{Ca}$; $0.35 \mathrm{Mg}$ and $1.85 \% \mathrm{~K}, \mathrm{DM}) \cdot \mathrm{d}^{-1}$. Cows were fed 1 $\mathrm{kg}$ of concentrate $(0.9 \mathrm{Ca} ; 0.42 \mathrm{Mg}$ and $1.38 \%$ $\mathrm{K}, \mathrm{DM}) / 2.5$ to $3.0 \mathrm{~kg}$ of milk during lactation. Blood samples were taken from the coccygeal vessels at peripartum (calving $\pm 1 \mathrm{~d}$ ) and analyzed for $\mathrm{Ca}$ and $\mathrm{Mg}$. The blood thresholds to classify cows as clinically or sub clinically hypocalcemic were: less than 5.5 and 5.5 to $8.0 \mathrm{mg}^{-\mathrm{dl}^{-1}}$ of $\mathrm{Ca}$, respectively. A value of $1.8 \mathrm{mg} \cdot \mathrm{dl}^{-1}$ of $\mathrm{Mg}$, or lower, was set to classify cows as hypomagnesemic. Although no significant differences

\footnotetext{
* Centro de Investigaciones en Nutrición Animal y Escuela de Zootecnia, Universidad de Costa Rica.
} 
difirió entre razas $(p \leq 0,05)$, las vacas Jersey tienden a tener valores menores de este mineral, especialmente al aumentar el número de partos. $\mathrm{La}$ prevalencia de hipomagnesemia difirió $(\mathrm{p}<0,05)$ entre las razas Jersey y Guernsey. La baja prevalencia de este desbalance sugiere que los casos de hipocalcemia clínica y subclínica observados se deben a aspectos biológicos propios de la homeostasis del Ca y no a mecanismos en la fisiología de este elemento en que interviene el Mg.

\section{INTRODUCCIÓN}

Los desbalances en el metabolismo del Ca que ocurren durante el periparto pueden ser muy onerosos para la producción del ganado lechero. Cuando los niveles de este elemento caen a niveles inferiores a aquellos necesarios para mantener la función nerviosa y muscular los animales no son capaces de mantenerse de pie y caen, condición que se denomina fiebre de leche o hipocalcemia. Si la reducción en el contenido de Ca no es tan drástica pero es inferior a los valores fisiológicamente normales, la vaca sufre de hipocalcemia subclínica, situación que causa una reducción en el consumo de alimentos, pobre motilidad del rumen e intestino, baja producción y reproducción y mayor susceptibilidad a otras enfermedades metabólicas e infecciosas. (Curtis et ál. 1983, Goff 2000, Goff 2006, Goff et ál. 2005). La prevalencia de este desbalance en el metabolismo del $\mathrm{Ca}$ es variable en los hatos de ganado lechero y se asocia con la raza, edad y la composición mineral de dieta (NRC 2001, Sánchez y Goff 2006). La prevalencia de fiebre de leche es mayor en los animales de las razas Jersey y Guernsey que los Holstein (Amaral 2014), así como en aquellos que han tenido un mayor número de partos. Goff et ál. (1995) han develado que las vacas Jersey son $(p \geq 0.05)$ were found among breeds for plasma $\mathrm{Ca}$ content at parturition, Jersey cows tend to have a lower content of this mineral, specially as parity number increases. Hipomagnesemia prevalence differed $(\mathrm{p}<0.05)$ between Jersey and Guernsey breeds. The low prevalence of this metabolic imbalance suggest that the clinical and subclinical hipocalcemia cases observed in this study due to biological aspects proper to $\mathrm{Ca}$ homeostasis and are not linked to Ca homeostatic mechanisms in which $\mathrm{Mg}$ participates.

más susceptibles a sufrir hipocalcemia que las Holstein por tener un menor número de receptores a la hormona 1,25 dihidroxivitamina D. Así mismo, Horst et ál. (1990) encontraron que las vacas adultas tienen una menor cantidad de los receptores antes indicados a nivel óseo e intestinal que las jóvenes, lo que hace que los animales de 3 o más partos sean más susceptibles a este desbalance metabólico. Otros autores (Liesegang et ál. 1998) amplían esta lista de factores y señalan que la producción de leche, condición corporal, otras enfermedades, clima e instalaciones en que se mantienen los animales también son factores de riesgo.

De igual manera, cantidades bajas de magnesio en la sangre o sea hipomagnesemia pueden afectar el metabolismo del ganado lechero. Esta reducción en el contenido de magnesio sanguíneo puede deberse al suministro de cantidades bajas de este mineral, al suministro de fuentes de magnesio poco biodisponibles o bien a la presencia a factores antagónicos a la absorción del mismo en la dieta, tales como el potasio. La reducción en el contenido de magnesio sanguíneo puede ser severa, presentándose el denominado síndrome de la vaca caída. Sin embargo, lo más común es que la hipomagnesemia sea moderada y en estos casos los niveles bajos de magnesio interfieren con 
la homeostasis del calcio, produciéndose una hipocalcemia secundaria a la hipomagnesemia (Goff 2006, Lean et ál. 2006).

Tanto la deficiencia de $\mathrm{Ca}$ como la de $\mathrm{Mg}$ afectan la contracción muscular, por lo que la sintomatología asociada a la hipocalcemia, la hipomagnesemia o la hipocalcemia secundaria a la hipomagnesemia, se caracterizan por movimientos atípicos al caminar, recumbencia, convulsiones, tetania y caída de los animales, los cuales responden positivamente a la terapia con ambos minerales. Por lo general los tratamientos para animales que después del parto muestran la sintomatología indicada contienen $\mathrm{Ca}, \mathrm{Mg}$ y glucosa (Goff y Horst 1993).

Las cantidades altas de potasio en la dieta interfieren con la absorción del $\mathrm{Mg}$ a nivel ruminal, pudiéndose presentar casos de hipomagnesemia en el ganado lechero aunque la dieta le aporte al animal las cantidades de $\mathrm{Mg}$ requeridas para satisfacer sus necesidades fisiológicas. Martens y Kasebieter (1983) encontraron que las concentraciones altas de potasio en el líquido ruminal despolarizan la membrana apical de las papilas ruminales, que reducen el potencial electromotriz requerido para que el $\mathrm{Mg}$ traspase la pared del rumen y se incorpore al metabolismo. Lo anterior hace que la nutrición del $\mathrm{Mg}$ en la vaca lechera sea muy sensible en aquellos sistemas de producción que hacen un uso muy intensivo de las pasturas, por cuanto contenidos del orden de 3,5\% de la materia seca son comunes en los pastos suculentos del trópico (Sánchez 2008).

El objetivo de esta investigación fue analizar la prevalencia de hipocalcemia clínica y subclínica y la hipomagnesemia en un hato conformado por vacas de las razas Holstein, Jersey y Guernsey, con una distribución muy similar en el número de animales por raza. Todas las vacas conviven en la misma finca, están sometidas a las mismas prácticas de manejo animal y sanitarias, pastorean en los mismos potreros y reciben el mismo alimento balanceado durante los períodos de vaca próxima al parto y de lactancia. La información obtenida en esta investigación permitirá establecer las posibles diferencias entre razas a fin de desarrollar prácticas de manejo nutricional que contribuyan a prevenir de una manera más asertiva la prevalencia de este desbalance metabólico según el origen racial del animal. Lo anterior en sistemas de producción de leche que se basan en el manejo intensivo de forrajes en ambientes tropicales.

\section{MATERIALES Y MÉTODOS}

El estudio se realizó en una finca de ganado lechero ubicada en el cantón de Oreamuno, Cartago, Costa Rica (9 $9^{\circ} 53^{\prime} 55^{\prime \prime}$ Latitud Norte, 8349'31" Longitud Oeste, 1700 m de altitud), durante el período comprendido entre enero y setiembre de 2012. El hato está constituido por vacas de las razas Holstein, Guernsey y Jersey, las cuales están sometidas a las mismas condiciones ambientales, prácticas de alimentación, manejo animal y sanitario y pastorean en un sistema rotacional intensivo de pasto kikuyo (Kikuyuocloa clandestina) (14,8\% MS, 23,4\% PC, 54\% FDN, 1,30 Mcal de $\mathrm{EN}_{\mathrm{L}} / \mathrm{kg}$ de $\mathrm{MS}(1 \mathrm{X}), 0,35 \% \mathrm{Ca}, 0,31 \% \mathrm{Mg}$ y $3,50 \% \mathrm{~K}$ ).

Durante las 3 semanas previas a la fecha probable de parto, todas las vacas fueron suplementadas con 4 kg.animal ${ }^{-1}$.día ${ }^{-1}$ de alimento balanceado bajo en $\mathrm{Ca}(14 \%$ PC, $31 \%$ FDN, 1,5 Mcal de $\mathrm{EN}_{\mathrm{L}} / \mathrm{kg}(1 \mathrm{X}), 0,2 \% \mathrm{Ca} ; 0,42 \% \mathrm{Mg}$ y $1,38 \% \mathrm{~K}$ ) y $1 \mathrm{~kg} \cdot$ animal $^{-1}$.día ${ }^{-1}$ de heno (Digitaria decumbens Stent, cv. Transvala) $(82,3 \%$ MS, 5,1\% PC, 61,4\% FDN, 1,0 Mcal de $\mathrm{EN}_{\mathrm{L}} / \mathrm{kg}$ (1X), $0,35 \%$ Ca; $0,30 \%$ Mg y 1,8\% K)/d. Después del parto y durante toda la lactancia las vacas fueron alimentadas con $1 \mathrm{~kg}$ de alimento balanceado (18,6\% PC, 1,8 Mcal de $\mathrm{EN}_{\mathrm{L}} / \mathrm{kg}(3 \mathrm{X}), 0,90 \% \mathrm{Ca}$; $0,42 \% \mathrm{Mg}$ y $1,38 \% \mathrm{~K}$ ) por cada 2,5 a $3,0 \mathrm{~kg}$ de leche producida.

A todas las vacas que parieron durante el período de estudio se les tomó muestras de sangre de los vasos sanguíneos coccígeos durante el periparto (día del parto \pm 1 día). En total se muestrearon 152 animales (62 Jersey, 41 Guernsey y 49 Holstein), de los cuales el 28,3; 21,7; 20,4; 16,4 y 
$13,2 \%$ correspondieron al $1^{\circ}, 2^{\circ}, 3^{\circ}, 4^{\circ}$ y $5^{\circ}$ o más partos, respectivamente. El 61,2\% de las muestras fue tomada el día del parto, $4,6 \%$ el día previo y $34,2 \%$ el día posterior al mismo.

Las muestras se mantuvieron en refrigeración y para separar el suero sanguíneo se centrifugaron a $3500 \mathrm{rpm}$ durante $10 \mathrm{~min}$ a $4^{\circ} \mathrm{C}$. Posteriormente al suero se le determinó el contenido de Ca y Mg por medio de espectrofotometría de absorción atómica con un espectrofotómetro Perkin Elmer A. Analist 800 (Zettner y Seligson 1964).

La información productiva, reproductiva y sanitaria del hato se registró a traves del el software VAMPP Bovino 3.0, la cual se trasladó posteriormente a los programas estadísticos IBM $^{\circledR}$ SPSS $^{\circledR}$ Statistics (version 21; IBM Corp., Armonk, NY) e InfoStat 1.0 (Di Rienzo et ál. 2011) para el análisis estadístico. Se realizó un análisis descriptivo de los valores promedio de las variables de interés según raza, las mismas fueron: número de parto, concentración de $\mathrm{Ca}$ y $\mathrm{Mg}\left(\mathrm{mg} \cdot \mathrm{dl}^{-1}\right)$ en suero sanguíneo y la relación entre dichas concentraciones $\left(\mathrm{Ca} . \mathrm{Mg}^{-1}\right)$; según el parto en el que se midió la concentración de dichos minerales. De igual forma el análisis descriptivo de medias se realizó para las variables intervalo entre partos, duración del período seco, duración de la preñez, duración de la lactancia durante el ciclo productivo previo y posterior a ese parto, producciones de leche y grasa, sólidos y proteína láctea. La medida de variabilidad seleccionada para estas variables fue el intervalo de confianza al 95\%. También se realizaron análisis de correlación de Pearson y análisis de regresión lineal simple, posteriormente se efectuaron pruebas de comparación múltiple de Tukey, protegidas por un análisis de varianza (ANOVA) para identificar diferencias significativas entre medias. Dichas diferencia fueron declaradas cuando $\mathrm{p}<0,05$. El coeficiente de chance o 'Odds Ratio' (OR) se utilizó como medida de asociación para determinar la razón de probabilidad de sufrir hipocalcemia o hipomagnesemia según la raza de interés.

\section{RESULTADOS Y DISCUSIÓN}

\section{Análisis descriptivo de las variables objeto de estudio}

Para caracterizar las variables de desempeño productivo, calidad de la leche, índices reproductivos y manejo del hato se recopiló información registral de la base de datos VAMPP 3.0., la cual se presenta en el Cuadro 1. 


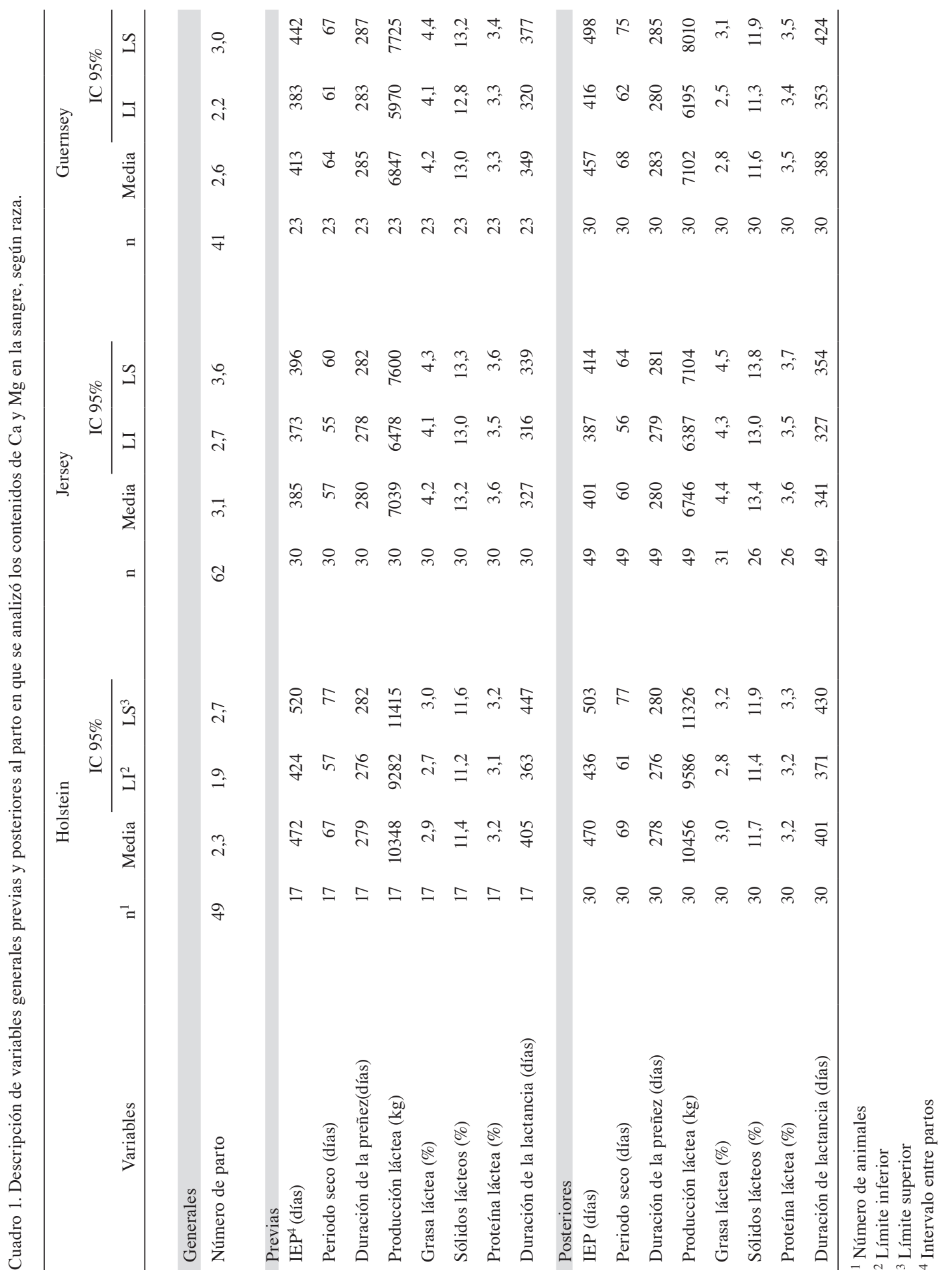


Efecto de la raza y número de parto sobre las concentraciones sanguíneas de Ca y $\mathrm{Mg}$

Las concentraciones promedio de $\mathrm{Ca}$ y $\mathrm{Mg}$ sanguíneos durante el periparto (día del parto \pm 1 día) en vacas de las razas Holstein, Jersey y Guernsey se presentan en el Cuadro 2.

Aunque no hubo diferencias significativas $(p \geq 0,05)$ entre los valores medios de Ca sanguíneo durante el periparto entre las razas estudiadas, los animales de la raza Jersey tuvieron los valores menores (7,49 mg.dl-1). Goff et ál. (1995) han encontrado que las vacas de la raza Jersey tienen una cantidad menor de receptores de la hormona 1, 25 dihidroxivitamina D $\left(95\right.$ fmol. $\mathrm{mg}^{-1}$ de proteína) que las vacas de la raza Holstein (142 fmol.mg ${ }^{-1}$ de proteína), lo que hace más proclives a las vacas Jersey a tener contenidos menores de calcio sanguíneo en momentos en que se reta el metabolismo de este elemento, como lo es el período de transición. En relación con el contenido de Mg sanguíneo, en la literatura no se asocia esta variable con la raza del animal. En este estudio se encontró que las vacas de la raza Jersey tienen cantidades de $\mathrm{Mg}$ sanguíneos mayores $(\mathrm{p}<0,05)$ que los animales de las razas Holstein y Guernsey. Lo anterior podría deberse a que todos los animales consumieron la misma cantidad de alimento balanceado durante el preparto $(4 \mathrm{~kg}$ de alimento con $0,42 \%$ de $\mathrm{Mg}$ por animal por día), y al ser éste el componente de la dieta que aporta la mayor cantidad de $\mathrm{Mg}$ y las vacas Jersey las de menor peso corporal y por consiguiente las que consumen menos materia seca (NRC 2001), el contenido ruminal tendrá una concentración mayor de Mg, lo cual según (Goff 2006) es determinante para que las vacas absorban $\mathrm{Mg}$ por la vía pasiva y que el animal incremente sus niveles de Mg sanguíneo.

Cuadro 2. Valores medios e intervalo de confianza al 95\% para la concentración de Calcio y Magnesio sanguíneo durante el periparto en vacas de las razas Holstein, Jersey y Guernsey en pastoreo.

\begin{tabular}{ccccc}
\hline & \multicolumn{2}{c}{$\mathrm{Ca}\left(\mathrm{mg} . \mathrm{dl}^{-1}\right)$} & \multicolumn{2}{c}{$\mathrm{Mg}\left(\mathrm{mg}^{\left.-1 l^{-1}\right)}\right.$} \\
Raza & Media & IC 95\% & Media & IC 95\% \\
\hline Holstein & 7,85 & $7,42-8,28$ & $2,37^{\mathrm{a}}$ & $2,22-2,53$ \\
Jersey & 7,49 & $7,11-7,87$ & $2,78^{\mathrm{b}}$ & $2,64-2,92$ \\
Guernsey & 8,09 & $7,63-8,56$ & $2,35^{\mathrm{a}}$ & $2,18-2,52$ \\
\hline
\end{tabular}

a,b Medias con letras diferentes en una misma columna difieren significativamente entre sí ( $<<0,001)$, según la prueba de Tukey.

Al analizar el efecto del número de parto sobre la concentración de Ca sanguíneo, se observó que la concentración de este mineral disminuye conforme aumenta el número de parto. Horst et ál. (1990) han explicado esta tendencia metabólica al encontrar que la concentración de los receptores de la hormona
1, 25 dihidroxivitamina $\mathrm{D}$ del intestino y los huesos disminuye con la edad del individuo. En la Figura 1 se observa que esta tendencia se acentúa aún más en los animales de la raza Jersey, con 5 o más partos concentraciones de Ca sanguíneo menores $(\mathrm{p}<0,05)$ que los de 2 partos. 


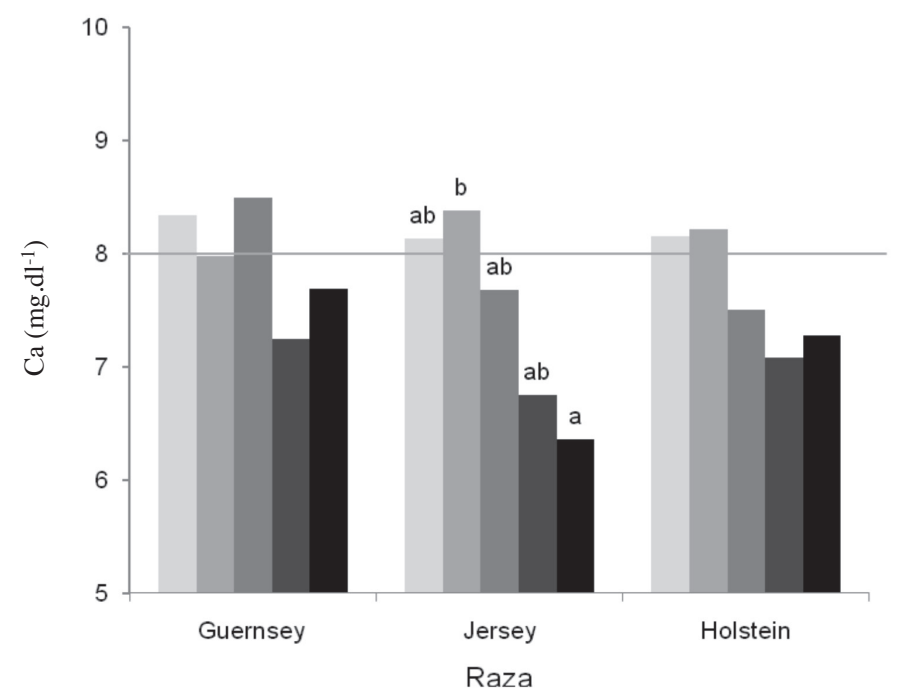

Fig. 1. Concentración de Ca sanguíneo en vacas de las razas Guernsey, Jersey y Holstein en pastoreo según el número de parto $(1,2,3,4$ y 5 o más partos en orden de aparición). Columnas con distinta letra dentro de una misma raza difieren entre sí $(\mathrm{p}<0,05)$ según la prueba de Tukey.

La concentración promedio de Ca sanguíneo en los animales de las razas Jersey y Holstein cae consistentemente por debajo de los $8 \mathrm{mg} \cdot \mathrm{dl}^{-1}$ después del segundo parto, mientras que en las vacas Guernsey esto ocurre después del tercero. Esta relación entre el número de partos y la prevalencia de hipocalcemia ha sido reportada previamente por (Reinhardt et ál. 2011, Sánchez y Saborío 2013). El análisis de las concentraciones de $\mathrm{Mg}$ en sangre no difirió según el número de parto en ninguna de las razas.

\section{Prevalencia de hipocalcemia e hipomagnesemia según raza}

Según Curtis et ál. (1983), uno de los retos que tiene la vaca durante el periparto es mantener la normocalcemia $\left(8,5\right.$ a $\left.10 \mathrm{mg}_{\mathrm{dl}} \mathrm{Cl}^{-1}\right)$; o por lo menos lograr que el grado de hipocalcemia propio de este período sea leve; ya que niveles de Ca en la sangre inferiores a $8,0 \mathrm{mg}^{-\mathrm{dl}^{-1}}$ pueden aumentar la ocurrencia de partos distócicos, retención de placenta, metritis, mastitis, desplazamiento del abomaso y cetosis; desórdenes que pueden afectar directa o indirectamente la producción de leche y la reproducción. Una concentración sanguínea inferior a $2 \mathrm{mmol}^{\mathrm{l}^{-1}} \mathrm{de}$ $\mathrm{Ca}\left(8,0 \mathrm{mg} \cdot \mathrm{dl}^{-1}\right)$, pero superior a $1,4 \mathrm{mmol} . \mathrm{l}^{-1}(5,5$ $\left.\mathrm{mg} . \mathrm{dl}^{-1}\right)$ se considera como hipocalcemia subclínica (NRC 2001), mientras que valores inferiores a 1,4 mmol. $\mathrm{l}^{-1}\left(5,5 \mathrm{mg} \cdot \mathrm{dl}^{-1}\right)$ definen una hipocalcemia clínica (Roche y Berry 2006). Por medio de los valores umbrales anteriores se obtuvo una prevalencia general de hipocalcemia de 56,58\% $(n=86)$. El 6,58\% fueron casos clínicos y el 50\% subclínicos. Según Goff (2008) la incidencia de fiebre de leche reportada en los hatos confinados es de 5 a 7\%, valores similares a los reportados por Roche (2003), quien indica que valores de prevalencia de $5 \%$ de hipocalcemia clínica son frecuentes en sistemas pastoriles. La prevalencia de hipocalcemia clínica encontrada en el presente estudio $(6,58 \%)$ es similar a los valores mencionados por los autores citados.

La prevalencia de hipocalcemia subclínica develada en este trabajo (50\%) sobrepasó la reportada por Reinhardt et ál. (2011), quienes en un estudio muy amplio en hatos confinados que involucró 1462 animales distribuidos en 480 hatos mayoritariamente de la raza Holstein 
obtuvieron una prevalencia de hipocalcemia subclínica superior al $40 \%$. Lo cual podría deberse a que las dietas en los sistemas pastoriles son más alcalinogénicas que en los sistemas estabulados
(Goff et ál. 2005) y a que en esta investigación involucra un número importante de animales Jersey. La prevalencia de hipocalcemia según raza se denota en el Cuadro 3.

Cuadro 3. Prevalencia de hipocalcemia e hipomagnesemia en animales en pastoreo de las razas Holstein, Jersey y Guernsey.

\begin{tabular}{|c|c|c|c|c|}
\hline \multirow[t]{2}{*}{ Raza } & \multirow[t]{2}{*}{ Población } & \multicolumn{2}{|c|}{ Hipocalcemia, n (\%) } & \multirow[t]{2}{*}{ Hipomagnesemia, n (\%) } \\
\hline & & Clínica & Subclínica & \\
\hline Holstein & 49 & $2(4)$ & $27(55)$ & $0(0)$ \\
\hline Jersey & 62 & $8(13)$ & $31(50)$ & 1 (2) \\
\hline Guernsey & 41 & $0(0)$ & $18(44)$ & $5(12)$ \\
\hline
\end{tabular}

En relación con el $\mathrm{Mg}$, la hipomagnesemia fue definida como valores inferiores a $1,8 \mathrm{mg}^{-1 l^{-1}}$ de Mg (Goff 2008). Según este criterio, la prevalencia general fue de 3,95\% (6 animales de 152 estudiados), asimismo en el Cuadro 3 se detalla la prevalencia de este desbalance metabólico por raza.

\section{Asociaciones relativas entre razas y entre otras variables}

Para determinar la asociación relativa entre razas en relación con la prevalencia de hipocalcemia e hipomagnesemia, se determinó el cociente de chance o 'Odds Ratio'; como también se les denomina; así como sus intervalos de confianza. El cociente de chance es una medida de asociación que indica la razón de la probabilidad de ocurrencia de un evento en relación con la no ocurrencia del mismo. Esta medida asocia una exposición (en este caso la raza del animal) con una respuesta (enfermedad) (Szumilas 2010). En este estudio se excluyó del análisis de asociación de hipocalcemia la raza Guernsey, así mismo la raza Holstein fue excluida del análisis de hipomagnesemia debido a que no hubo casos de estos desbalances metabólicos en dichas razas. En el Cuadro 4 se denotan las medidas de asociación indicadas.

Cuadro 4. Hipocalcemia e hipomagnesemia durante el peri-parto según raza en un sistema de producción de leche basado en el uso intensivo de pastos tropicales.

\begin{tabular}{ccccccc}
\hline & \multicolumn{2}{c}{ Positivas } & \multicolumn{2}{c}{ Negativas } & OR* & IC 95\% \\
& $\mathrm{n}$ & $\%$ & $\mathrm{n}$ & $\%$ & & \\
\hline Hipocalcemia & 2 & 4 & 47 & 96 & 1 & $0,76-15,9$ \\
Holstein & 8 & 13 & 54 & 87 & 3,48 & \\
Jersey & & & & & & \\
& 1 & 2 & 61 & 98 & 1 & $1,30-55,2$ \\
\hline Hipomagnesemia & 5 & 12 & 36 & 88 & 8,47 & \\
Jersey & & & & & & \\
Guernsey & & & & & & \\
\hline
\end{tabular}

*Odds Ratio. 
El análisis del coeficiente de chance (OR) sugiere que las vacas de la raza Jersey pueden ser más propensas a sufrir fiebre de leche que las de la raza Holstein; sin embargo, según el intervalo de confianza $(0,76$ a 15,9$)$ ambas razas tienen la misma susceptibilidad a sufrir este desbalance metabólico. Lo anterior podría deberse al tamaño de la muestra analizada, ya que la literatura indica que las vacas de la raza Jersey tienen una cantidad menor de receptores de la hormona 1, 25 dihidroxivitamina $\mathrm{D}$ que las vacas de la raza Holstein, lo cual hace que la homeostasis del calcio en las vacas Jersey sea más vulnerable y por lo tanto más proclives a padecer este desbalance metabólico durante el período de transición (Goff et ál. 1995).

En relación con la susceptibilidad a la hipomagnesemia, en el hato objeto de estudio las vacas Guernsey podrían ser más susceptibles a este desbalance metabólico que las Jersey. En la literatura no existen evidencias científicas que respalden estos hallazgos, sin embargo los balances nutricionales (NRC 2001) sugieren que las vacas de la raza Jersey en este hato consumen una cantidad de magnesio mayor que las de la raza Guernsey. Lo anterior debido a que las vacas de ambas razas consumen la misma cantidad de alimento balanceado antes del parto $(4 \mathrm{~kg}$ por animal por día), y éste a su vez contiene más magnesio que los forrajes ( 0,42 vs. $0,31 \%$ de la materia seca), los cuales son el complemento de la ración consumida.

Al realizar análisis de correlaciones y regresiones lineales se encontraron asociaciones significativas $(\mathrm{p}<0,05)$ entre la concentración de Ca sanguínea y variables de desempeño productivo y reproductivo, las cuales se denotan en el Cuadro 5.

Los resultados que se presentan en el Cuadro 5 indican que las asociaciones encontradas son tendencias, en la mayoría de los casos. Esto podría deberse a que el número de animales para cada grupo racial es relativamente pequeño $(\mathrm{n}<63)$. Sin embargo, la significancia estadística sugiere que la tendencia observada podría mantenerse constante al incrementar el número de vacas en la muestra.

Cuadro 5. Coeficientes de correlación de Pearson y regresión lineal entre variables asociadas a la concentración de Ca sanguíneo durante el peri-parto en vacas en pastoreo intensivo, según raza.

\begin{tabular}{|c|c|c|c|c|}
\hline \multirow[b]{2}{*}{ Variable } & \multicolumn{4}{|c|}{ Concentración de Ca (mg.dl $\left.{ }^{-1}\right)$ sanguíneo como variable de asociación } \\
\hline & $\begin{array}{l}\text { Correlación de } \\
\text { Pearson }\end{array}$ & Regresión lineal & Valor $\mathrm{p}$ & Raza \\
\hline & Rho & $\mathrm{R}^{2}$ & $\mathrm{P}$ & \\
\hline Producción láctea previa** & $-0,516$ & 0,266 & 0,012 & Guernsey \\
\hline Producción láctea posterior* & $-0,371$ & 0,138 & 0,043 & Guernsey \\
\hline $\mathrm{CCC}$ al parto** & $-0,312$ & 0,097 & 0,024 & Jersey \\
\hline Número de parto** & $-0,335$ & 0,112 & 0,008 & Jersey \\
\hline Número de parto** & $-0,323$ & 0,085 & 0,024 & Holstein \\
\hline IEP posterior* & $-0,403$ & 0,163 & 0,027 & Holstein \\
\hline
\end{tabular}

*Variable dependiente

***ariable Independiente 
La mayor asociación encontrada entre las variables descritas en el Cuadro 5 fue entre la producción láctea previa al parto y la concentración de $\mathrm{Ca}$ en la sangre durante el peri-parto en las vacas Guernsey, donde se determinó que el 26,6\% de la concentración de Ca durante este período se explica por dicha producción. Así mismo, el intervalo entre partos; que es una variable que tiene un impacto significativo sobre la rentabilidad de los hatos de ganado lechero, se explica en un 16,3\% por la concentración de Ca sanguíneo durante el peri-parto en vacas de la raza Holstein. El comportamiento reproductivo del ganado lechero es la manifestación de múltiples factores fisiológicos y ambientales, entre los cuales está la hipocalcemia clínica y subclínica que afecta directa o indirectamente (por medio de enfermedades metabólicas e infecciosas asociadas) en forma negativa los índices reproductivos en el ciclo reproductivo posterior al parto (Wilde 2006).

\section{CONCLUSIONES}

La prevalencia de hipocalcemia clínica documentada en el hato de ganado lechero objeto de estudio es similar a la reportada en la literatura para hatos estabulados; no ocurriendo lo mismo para la hipocalcemia subclínica; la cual fue mayor en este hato que pastorea forrajes suculentos con 3,5\% de potasio de la materia seca y consume una dieta total con un valor estimado de $2,7 \%$ de este mineral, el cual determinante en la prevalencia de la hipocalcemia. El número de partos de las vacas juega un papel importante en la prevalencia de la hipocalcemia, acentuándose esta condición significativamente $(\mathrm{p} \leq 0,05)$ en las vacas de la raza Jersey. Aunque no hubo diferencias significativas entre razas para el contenido de Ca sanguíneo durante el periparto, se encontró que las vacas Jersey tienden a tener valores menores de este mineral, lo cual afecta negativamente el desempeño productivo y reproductivo de los animales. La baja prevalencia de hipomagnesemia encontrada en este estudio sugiere que los casos de hipocalcemia clínica y subclínica observados se deben especialmente a aspectos biológicos propios de la homeostasis del $\mathrm{Ca}$ en el animal y no a mecanismos en la fisiología del $\mathrm{Ca}$ en que interviene el Mg.

\section{AGRADECIMIENTO}

Los autores expresan su agradecimiento al Sr. Oscar Martínez Segura propietario de la finca Los Espinos, en Cipreses de Oreamuno, Cartago por facilitar el hato de ganado lechero donde se realizó este estudio. Asimismo, al Médico Veterinario Andrés Martínez Araya y al Sr. Luis Fernando Guillén Fernández por la colaboración ofrecida durante la realización de esta investigación.

\section{LITERATURA CITADA}

AMARAL D. 2014. Subclinical hypocalcemia, or milk fever, in dairy cows- why all the fuss? Americas Research-based Learning Network. Consultado el 15 de mayo de 2014. Disponible en https:// www.extension.org/pages/70227/subclinicalhypocalcemia-or-milk-fever-in-dairy-cows-why-allthe-fuss\#.VA86W6NZhtg

BALZARINI M.G., GONZALEZ L., TABLADA M., CASANOVES F., DI RIENZO J.A., ROBLEDO C.W. 2008. Manual del Usuario, Editorial Brujas, Córdoba, Argentina. 336 p.

CURTIS C.R., ERB H.N., SNIFFEN G.J., SMITH R.D., POWERS P.A., SMITH M.C., WHITE M.E., HILLMAN R.B., PEARSON E.J. 1983. Association of parturient hypocalcemia with periparturient disorders in Holstein cows. J. Am. Vet. Med. Assos. 183:559-561.

Di RIENZO J.A., CASANOVES F., BALZARINI M.G., GONZALEZ L., TABLADA M., ROBLEDO C.W. 2011. InfoStat versión 2011. Grupo InfoStat, FCA, Universidad Nacional de Córdoba, Argentina. Consultado el 10 de junio del 2014 Disponible en http://www.infostat.com.ar

GOFF J.P. 2000. Pathopysiology of calcium and phosphorus disorders. Vet. Clin. North Am. Food Anim. Pract. 13:619-639.

GOFF J.P. 2006. Macromineral physiology and application to thefeeding of the dairy cow for prevention of milkfever and other periparturient mineral disorders. Animal Feed Science and Technology 126:237-257.

GOFF J.P. 2008. The monitoring prevention and treatment of milk fever and subclinical hipocalcemia in dairy cows. Vet. J. 176:50-57. 
GOFF J.P., HORST R.L. 1993. Oral administration of calcium salts for treatment of hypocalcemia in cattle. J. Dairy Sci. 76:101-108.

GOFF J.P., REINHARDT T.A., BEITZ D.C., HORST R.L. 1995. Breed effects tissue vitamin D receptor concentration in periparturient dairy cows: a milk fever risk factor? J. Dairy Sci. 78 (1):184.

GOFF J.P., SÁNCHEZ J.M., HORST R.L. 2005. Hypocalcemia biological effects and strategies for prevention. Mineral Nutrition Conference.University of Tennessee. USA. $6 \mathrm{p}$.

HORST R.L. 1986. Regulation of calcium and phosphorus homeostasis in the dairy cow. J. Dairy Sci. 69:604616.

HORST R.L., GOFF J.P., REINHARDT T.A. 1990. Advancing age results in reduction of intestinal and bone 1, 25 dyhidroxyvitamin D receptor. Endocrinology 126:1053-1057.

HORST R.L., GOFF J.P., REINHARDT T.A. 1994. Calcium and vitamin D metabolism in the dairy cow. J. Dairy Sci. 77:1936-1951.

LEAN I.J., DeGARRIS P.J., McNEIL D.M., BLOCK E. 2006. Hipocalcemia in dairy cows: Meta-analysis in dietary cation-anion difference theory revisited. J. Dairy Sci. 89:669-684.

LIESEGANG A., SASSI M.L., RISTELI J., EICHER R., WANNER M., RIOND. 1998. Comparison of bone resorption markers during hypocalcemia in dairy cows. J. Dairy Sci. 81:2614-2622.

MARTENS H., KASEBIETER H. 1983. In vitro studies of the effect of sodium and potassium ions on magnesium transport across the isolated rumen mucosa of sheep. Zentralbl Veterinarmed (A) 30:1-14.

NATIONAL RESEARCH COUNCIL 2001. Nutrient Requirements of dairy Cattle. $7^{\text {th }}$ rev. Ed. National Academy Press. Washington, D. C. 381 p.

REINHARDT T.A., LIPOLIS J.D., McCLUSKEY B.J., GOFF J.P., HORST R.L. 2011. The Veterinary Journal 188:122-124.

ROCHE J.R. 2003. The incidence and control of hipocalcemia in pasture-based systems. Acta Vet. Scand. 97:141144.

ROCHE J.R., BERRY D.P. 2006. Periparturient climatic, animal, and management factors influencing the incidence of milk fever in grazing systems. J. Dairy Sci. 89:2775-2783.

SÁNCHEZ J.M. 2008. El pasto kikuyo y su aporte a la nutrición de vacas lecheras, pp. 137-155. In: VI Seminario Internacional. Competitividad en carne y leche. Medellín, Colombia.

SÁNCHEZ J.M., SABORÍO A. 2013 Prevalence of hypocalcemia in grazing Jersey herds in Costa Rica. (Abstract) International Conference in Production Disease in Farm Animals, Uppsala, Sweden, pp. 150.

SZUMILAS M. 2010. Explaining Odds Ratios. J. Can. Acad. Child Adolesc Psychiatry 19(3):227-229.

WILDE D. 2006. Influence of macro and micro minerals in the peri-parturient period on fertility in the dairy cattle. Animal Reproduction Science 96:240-249.

ZETTNER A., SELIGSON D. 1964. Application of atomic absorption spectrophotometry in the determination of calcium in serum. Atomic Absorption Spectrophotometry 10(2):869-890. 
